\title{
Variação no número de glândulas e produção de óleo em flores de Stigmaphyllon paralias A. Juss. (Malpighiaceae)
}

\author{
Paula Dib de Carvalhoº ${ }^{1}$ Eduardo Leite Borba ${ }^{1,3}$ e Angélica Maria Lucchese ${ }^{2}$
}

Recebido em 04/11/2003. Aceito em 20/08/2004

\begin{abstract}
RESUMO - (Variação no número de glândulas e produção de óleo em flores de Stigmaphyllon paralias A. Juss. (Malpighiaceae)). Foi estudada uma população de Stigmaphyllon paralias (Malpighiaceae) em Feira de Santana, Bahia, Brasil, visando analisar a variação do número de glândulas e sua produção de óleo nas flores. Dos indivíduos analisados, 76\% não variaram o número de glândulas entre suas flores, ocorrendo 41\% de indivíduos com flores que possuem 10 glândulas, 31\% com oito glândulas e 4\% com nove glândulas. Nas flores dos morfos que apresentam oito e nove glândulas, as glândulas ausentes são sempre as da sépala inferior. Centris leprieuri e Epicharis sp. (Apidae: Centridini) foram as únicas espécies que visitaram S. paralias, coletando óleo como recompensa. A sépala inferior localiza-se sob o abdome do polinizador quando pousado na flor, tornando-se inacessível à coleta de óleo. Sugerimos que a incapacidade de utilização de algumas glândulas pelos polinizadores possa ter possibilitado o aparecimento de morfos nas populações que não apresentam estas glândulas como caráter adaptativo por gerar economia de recursos na produção de recompensa não utilizada pelo polinizador. Todas as glândulas de todos os morfos são funcionais. As flores dos dois morfos principais com oito e 10 glândulas, produzem a mesma quantidade total de óleo. As glândulas das flores com oito glândulas aumentam a produção em cerca de $20 \%$, compensando a produção total de óleo por flor.
\end{abstract}

Palavras-chave: Centridini, glândulas de óleo, polinização

ABSTRACT - (Variation in the number of glands and oil production in flowers of Stigmaphyllon paralias A. Juss. (Malpighiaceae)). A population of Stigmaphyllon paralias (Malpighiaceae) was studied in Feira de Santana, Bahia, Brazil. Variation of the number of oil glands and oil production in the flowers were analized. $76 \%$ of the individuals did not present variation on the number of glands among their flowers, $41 \%$ presented flowers with 10 glands, 31\% with eight glands and $4 \%$ with nine glands. In the flowers of the morphs with eight or nine glands, the absent glands were always associated to the inferior sepal. Only Centris leprieuri and Epicharis sp. (Apidae: Centridini) visited flowers of $S$. paralias, collecting oil as rewarding. The inferior sepal is located under the pollinator abdomen when it is standing in the flower, therefore being inaccessible for oil collection. We suggest that the occurrence of glands not utilized by pollinators has allowed the appearance of morphs in the populations that do not present those glands. All glands of all flower morphs are functional. Flowers of the two more representative morphs, bearing eight and 10 glands, produce the same amount of oil. Glands of flowers with lower number of glands (eight) increase nearly $20 \%$ plus oil production, compensating oil production per flower.

Key words: Centridini, oil glands, pollination

\section{Introdução}

A família Malpighiaceae possui distribuição pantropical, incluindo cerca de 66 gêneros e 1.200 espécies, das quais aproximadamente $85 \%$ são neotropicais (Anderson 1979). No Brasil, ocorrem 34 gêneros e cerca de 300 espécies, distribuídas em formações vegetais diversas como florestas, cerrados, campos rupestres e caatingas (Barroso 1991).

As espécies neotropicais de Malpighiaceae, apesar de divergirem em vários aspectos como hábito, tipo de fruto, morfologia polínica e número cromossômico (Anderson 1979), tendem a ser relativamente uniformes quanto à morfologia floral, relacionada principalmente ao mecanismo de polinização (Anderson 1979; Sazima \& Sazima 1989). As flores da maioria das espécies neotropicais de Malpighiaceae produzem óleo em glândulas (elaióforos), comumente em número de 10, localizadas externamente ao cálice, sendo um par por sépala, e as pétalas são ungüiculadas, sendo uma delas modificada em cor e/ou espessura do unguículo, tendo por função atrair e sustentar o polinizador que se prende pela mandíbula ao visitar a flor. Este óleo é coletado por

\footnotetext{
1 Universidade Estadual de Feira de Santana, Departamento de Ciências Biológicas, Rodovia BR 116, km 03, CEP 44031-460, Feira de Santana, BA, Brasil

2 Universidade Estadual de Feira de Santana, Departamento de Ciências Exatas, Rodovia BR 116, km 03, CEP 44031-460, Feira de Santana, BA, Brasil

3 Autor para correspondência: borba@gmx.net
} 
abelhas fêmeas da tribo Centridini (Anthophoridae), sendo utilizado na alimentação e desenvolvimento de suas larvas e construção dos ninhos (Vogel 1990; Simpson \& Neff 1983; Buchmann 1987; Vinson et al. 1997). Por outro lado, no Velho Mundo, apenas 52\% das espécies de Malpighiaceae oferecem óleo aos polinizadores, podendo ser encontrado, nas outras espécies, pólen ou néctar como recompensa (Vogel 1990).

As abelhas da tribo Centridini são endêmicas do Novo Mundo e, de acordo com Vogel (1990), as características florais de Malpighiaceae americanas sofreram forte pressão seletiva destas abelhas coletoras de óleo. Segundo Anderson (1979), estas características resultam numa relação muito estreita entre estes dois grupos e, conseqüentemente, é observada pequena diversidade de polinizadores para as Malpighiaceae do Novo Mundo. Apesar disso, este sistema especializado foi decisivo para o grande sucesso das espécies de Malpighiaceae neotropicais em relação aos membros do Velho Mundo (Vogel 1990) e parece explicar a uniformidade floral destas espécies (Anderson 1979).

A ausência de glândulas de óleo em Malpighiaceae ocorre em alguns gêneros neotropicais, sendo esta uma condição derivada (Anderson 1979). Variação intragenérica tem sido observada em alguns gêneros neotropicais, como em Banisteriopsis Rob., com cerca de 92 espécies (Gates 1982), onde B. lutea (Griseb.) Cuatrec. não possui glândulas, mimetizando espécies simpátricas glandulosas que apresentam morfologia floral similar, como B. pubipetala (A. Juss.) Cuatrec. (Sazima \& Sazima 1989; M.R. Sigrist, dados não publicados).

Além de variação intragenérica na presença de elaióforos, variação intraespecífica também tem sido observada. Segundo Vogel (1990), algumas espécies do gênero Byrsonima Rich. são caracterizadas pela ausência de elaióforos em determinados indivíduos de uma mesma população, como Byrsonima sericea DC. (Teixeira \& Machado 2000). O mesmo foi observado por Sazima \& Sazima (1989) em Banisteriopsis muricata (Cav.) Cuatrec. e Heteropterys aceroides Griseb. Observações realizadas e evidências experimentais (Sazima \& Sazima 1989; Teixeira \& Machado 2000; M.R. Sigrist, dados não publicados) indicam a ocorrência de automimetismo em flores com e sem elaióforos destas três espécies e engano do polinizador, em função do modo de polinização.

Outro tipo de variação no número de elaióforos tem sido mencionado em espécies neotropicais que não possuem indivíduos completamente eglandulados. A ocorrência de poucas glândulas (1-3) é observada em várias espécies de Galphimia Cav. (Anderson 1979). C.B.N. Costa (dados não publicados) mencionou a variação no número de glândulas em Heteropterys alternifolia W.R. Anderson, que possui de sete a 10 glândulas e Stigmaphyllon paralias A. Juss., que possui de oito a 10 glândulas. Tem sido dada pouca atenção a este tipo de variação e estes autores não fizeram quaisquer suposições ou procuram demonstrar a natureza desta variação numérica em indivíduos.

Neste trabalho, realizou-se um estudo da variação do número de glândulas e da produção de óleo em flores em uma população de Stigmaphyllon paralias A. Juss. (Malpighiaceae) correlacionando os resultados com a biologia reprodutiva da espécie.

\section{Material e métodos}

A população de Stigmaphyllon paralias estudada localiza-se no campus da Universidade Estadual de Feira de Santana, Bahia, localizada no "Polígono das Secas”, em região de clima semi-árido caracterizado por ter clima seco e com médias anuais de pluviosidade geralmente inferiores a $1.000 \mathrm{~mm}$. A área de estudo abrangeu toda a área não construída da Universidade, parcialmente antropizada. Os indivíduos de $S$. paralias ocorrem em toda a área de estudo e geralmente possuem distribuição agrupada. Esses agrupamentos ocorrem em pequenos trechos de Cerrado e caatinga, com pouca vegetação arbustiva e predominância de vegetação herbácea e solo arenoso.

Foram marcados 100 indivíduos para a análise da produção de inflorescências e flores, e do número e posição das glândulas de óleo (elaióforos) nas flores, para determinar a variação dentro da população e dentro do indivíduo.

Foram realizadas observações em campo de setembro/2002 a março/2003 para registrar o comportamento dos visitantes na flor: quais glândulas foram utilizadas, recurso utilizado (óleo ou pólen) e horário de visita. As observações foram realizadas inicialmente entre 6 e 17 horas, em observações de 15 minutos em cada hora, sendo depois ajustado de 7 às 11 horas e de 15 às 18 horas, sem pausas na observação.

Quantificou-se a produção de óleo dos morfos mais freqüentes para verificar se estes produzem a mesma quantidade de óleo total por flor. As flores foram ensacadas ainda em botão para evitar o contato com visitantes florais. Os elaióforos de 180 flores, sendo 108 com 10 glândulas e 72 com oito glândulas, foram 
extraídos com auxílio de estilete, na fase de antese, e colocados em dois frascos para separação. As flores foram agrupadas por morfo floral, uma vez que os elaióforos são muito pequenos para serem analisados separadamente. As glândulas foram inicialmente pesadas, sendo depois colocadas para dessecar utilizando sílica-gel. Foram novamente pesadas e, em seguida, foi feita a extração do óleo utilizando como solvente $10 \mathrm{ml}$ de pentano, sendo realizadas três extrações, uma a cada 24 horas. Ao final do procedimento, as glândulas foram novamente pesadas, sendo obtida a quantidade média de óleo produzido por flor em cada morfo.

Foram realizados testes com Sudan IV, corante que evidencia a presença de lipídios, para verificar a funcionalidade das glândulas em diferentes morfos florais. Colocaram-se algumas gotas de Sudan IV em
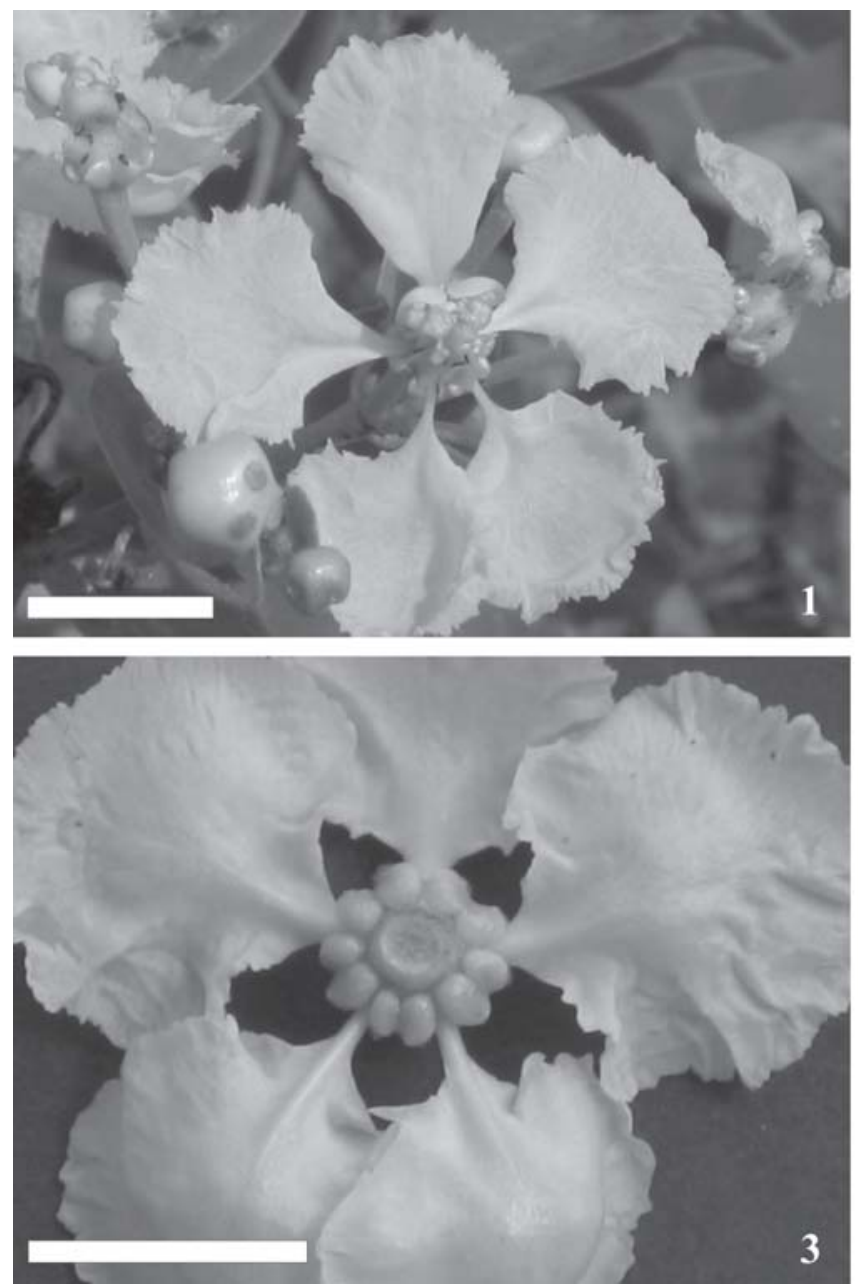

todas as glândulas de 70 flores de diferentes morfos, aguardou-se por cinco minutos e em seguida as glândulas foram lavadas em água corrente para retirar o excesso do corante. Foram feitas observações em estereomicroscópio para verificar quais glândulas tinham sido coradas.

\section{Resultados}

Os indivíduos da população estudada de Stigmaphyllon paralias são arbustos ou subarbustos, variando entre 30 a $150 \mathrm{~cm}$ alt. As flores possuem de 30 a 35 mm diâm., são levemente zigomorfas e estão dispostas em umbelas terminais (Fig. 1). O cálice possui cinco sépalas biglandulares. Essas glândulas são epiteliais, ovaladas, glabras, revestidas com uma fina cutícula onde o óleo é armazenado. A corola é composta
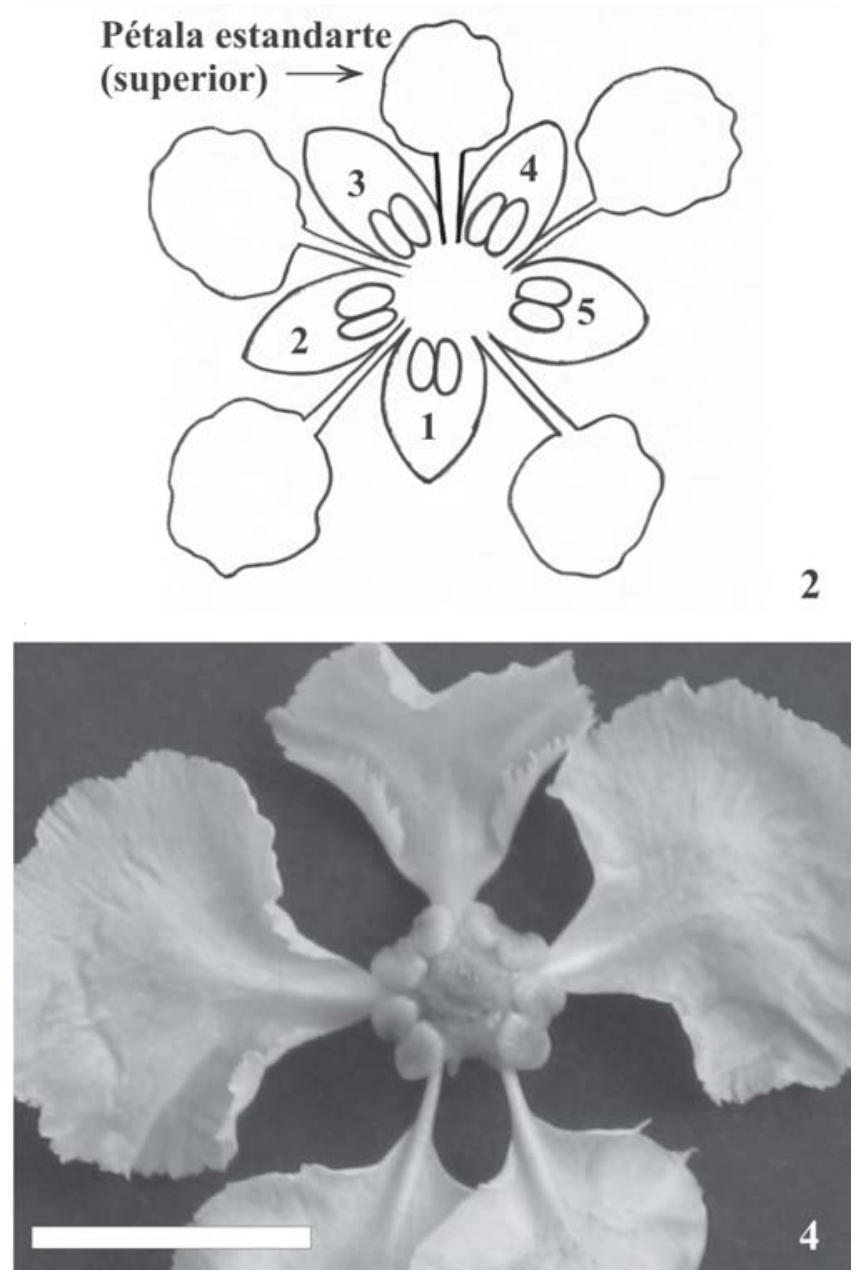

Figura 1. Flor de Stigmaphyllon paralias A. Juss., no campus da Universidade Estadual de Feira de Santana. Figura 2. Esquema de flor de S. paralias A. Juss., indicando o posicionamento das glândulas de óleo nas sépalas, e a numeração adotada neste trabalho. Figura 3-4. Vista inferior de flores dos morfos de S. paralias A. Juss. com 10 (3) e 8 (4) glândulas, este último faltando o par da sépala 1. Barras de escala $=10 \mathrm{~mm}$. 
por cinco pétalas patentes, amarelas, glabras, ungüiculadas, com cerca de $15 \mathrm{~mm}$ compr. e $15 \mathrm{~mm}$ larg., margem fimbriada e limbo pouco côncavo. A pétala superior ou estandarte possui o ungüículo mais espesso e limbo um pouco menor que as demais. Neste trabalho considerou-se a sépala em posição oposta à pétala superior como número 1 e no sentido horário foram todas numeradas de 1-5 (Fig. 2). O androceu é composto por 10 estames (cinco maiores e cinco menores). O gineceu apresenta três estiletes verdes com ápices modificados em forma de folha onde se encontram os estigmas que cobrem as anteras. $\mathrm{O}$ ovário é tricarpelar, trilocular, com um óvulo por lóculo.

Os indivíduos da população estudada floresceram durante todo o período de observação. O pico de floração ocorreu nos meses de fevereiro e março, coincidindo com um breve período de chuvas. Cada indivíduo apresentou de 1-6 inflorescências, sendo cada uma composta de 3-15 flores. A antese ocorreu entre 6-12 horas. Apenas 2-3 flores abriram por inflorescência num mesmo período de tempo, com duração da antese em torno de dois dias. A espécie estudada não apresentou mudança na coloração das pétalas após a antese, permanecendo todas amarelas.

Dos indivíduos analisados, $76 \%$ não variaram o número de glândulas entre as flores no mesmo indivíduo, sendo $41 \%$ de indivíduos com flores que possuem 10 glândulas, 31\% de indivíduos com flores que possuem oito glândulas e 4\% de indivíduos com flores que possuem nove glândulas (Fig. 3-4, Tab. 1).

Dos 24 indivíduos com variação no número de glândulas de óleo entre suas flores, 79,1\% $(\mathrm{n}=19)$ possuem um único morfo alternativo, podendo apresentar entre 4 e $29 \%$ das flores do segundo morfo (Tab. 1). Os demais 20,9\% ( $n=5)$ possuem três morfos no total, apresentando simultaneamente flores com oito, nove e 10 glândulas. Nos indivíduos que apresentam variação, aqueles com flores predominantemente com oito glândulas $(n=9)$ podem possuir também flores com nove $(66,7 \%$; $n=6)$ glândulas, principalmente, ou mais raramente flores com seis $(11,1 \% ; n=1)$ ou sete $(22,2 \% ; n=3)$ glândulas. Aqueles com flores predominantemente com nove glândulas podem possuir flores com oito $(66,7 \%$; $n=2)$ ou 10 glândulas (33,3\%; $\mathrm{n}=1)$. Todos indivíduos com predominantemente 10 glândulas e que variam ( $(n=3)$ possuem flores com nove glândulas. Quatro indivíduos apresentam dois morfos (oito e 10 glândulas) sem predominância de nenhum destes.

Em todas as flores que possuem apenas oito glândulas ( $\mathrm{n}=360)$, a ausência está relacionada à sépala 1 , assim como as flores que possuem nove glândulas, podendo neste caso faltar a glândula do lado direito (41\%, $n=26)$ ou do esquerdo (59\%, $n=38$ ). Nas flores com sete glândulas examinadas, a ausência relaciona-se ao par da sépala 1 e uma glândula na sépala 2 do lado direito, enquanto que na flor com seis glândulas, foi verificada ausência total na sépala 1 e parcial nas sépalas 2 e 5, permanecendo uma glândula central em cada uma destas sépalas. Não foi observada a ausência parcial ou total nas sépalas 3 e 4 que são superiores e próximas à pétala estandarte.

No campus da Universidade Estadual de Feira de Santana, Centris leprieuri e Epicharis sp. (Apidae: Centridini) foram as únicas espécies que visitaram S. paralias em todo o período de tempo de observação. As visitas foram muito raras e foi possível observar pouco em relação ao comportamento do polinizador, apesar da elevada frutificação observada. Em torno de 7 a 9 horas da manhã, as abelhas exercem

Tabela 1. Percentagem de ocorrência de indivíduos de Stigmaphyllon paralias A. Juss. com diferentes morfos florais, e percentagem de ocorrência de morfos alternativos em indivíduos que apresentam mais do que um morfo floral, no campus da Universidade Estadual de Feira de Santana, BA, Brasil, no período de setembro/2002 a março/2003.

\begin{tabular}{|c|c|c|c|c|c|c|}
\hline \multirow[t]{2}{*}{ Glândulas por flor } & \multirow[t]{2}{*}{$\begin{array}{l}\text { Indivíduos } \\
\quad(\%)\end{array}$} & \multicolumn{5}{|c|}{$\begin{array}{c}\% \text { de indivíduos com o morfo alternativo } \\
\text { (\% de flores do morfo alternativo entre parênteses) }\end{array}$} \\
\hline & & 6 glandulas & 7 glandulas & 8 glandulas & 9 glandulas & 10 glandulas \\
\hline oito & 31 & - & - & - & - & - \\
\hline nove & 4 & - & - & - & - & - \\
\hline 10 & 41 & - & - & - & - & - \\
\hline predominantemente 8 & 9 & $1(4 \%)$ & $2(10-25 \%)$ & - & $6(4-29 \%)$ & 0 \\
\hline predominantemente 9 & 3 & 0 & 0 & $2(7-8 \%)$ & - & $1(25 \%)$ \\
\hline predominantemente 10 & 3 & 0 & 0 & 0 & $3(14-17 \%)$ & - \\
\hline 8 ou 10 (50\% de cada morfo) & 4 & - & - & - & - & - \\
\hline 8 ou 9 ou 10 & 5 & - & - & - & - & - \\
\hline
\end{tabular}


comportamento de forrageio sem coleta de óleo. Após esse horário, elas saem para a coleta do óleo em várias flores de $S$. paralias. Para realizar a coleta do óleo, as abelhas pousam sobre a flor, contactando os estigmas e estames com a região ventral, com a cabeça sempre voltada para a pétala estandarte. Nesta posição, abraçam a flor e prendem a mandíbula no ungüículo da pétala estandarte, raspando os elaióforos com os dois primeiros pares de pernas para a extração do óleo, enquanto o último par fica estendido. Em seguida, quando em vôo, transferem esse recurso para a escopa do último par de pernas. A coleta do óleo pelas abelhas nas flores tem duração de poucos segundos.

Foi encontrado o total de 12,8 mg de óleo em flores com 10 glândulas, perfazendo a média de 0,119 mg de óleo por flor e 0,011 mg por glândula. Nas flores com oito glândulas foi encontrado o total de 8,2 mg de óleo com a média de $0,114 \mathrm{mg}$ por flor e $0,0143 \mathrm{mg}$ por glândula.

O teste de funcionalidade dos elaióforos realizado com 70 flores de diferentes morfos, feito com Sudan IV, revelou que todos os pares de glândulas são funcionais, uma vez que houve coloração avermelhada, indicando a presença de lipídios nestes.

\section{Discussão}

De acordo com Vogel (1990), a presença de ungüículo nas pétalas e o arranjo destas na flor são importantes características, pois determinam certo espaço entre cada pétala, que é utilizado pelas abelhas polinizadoras para encaixar as pernas e acessar os elaióforos para coletar o óleo. A disposição dos estames e estiletes no centro da flor de $S$. paralias favorece a polinização, pois possibilita aos polinizadores contato simultâneo com as anteras e os estigmas durante a coleta de óleo (C.B.N. Costa, dados não publicados).

No presente trabalho, $S$. paralias apresentou um índice muito pequeno de diversidade de polinizadores e de visitas, apesar da grande oferta de flores. Segundo C.B.N. Costa (dados não publicados), Byrsonima microphylla e $S$. paralias são as espécies mais abundantes em dunas na região de Salvador, Bahia, e apresentam os menores índices de diversidade e eqüitabilidade de visitantes. Entre as espécies de Malpighiaceae analisadas naquele trabalho, S. paralias é a com menor oferta de flores por indivíduo e apresenta baixa frequência de diversidade de visitantes, sendo polinizada por Centris flavifrons, com maior freqüência de visitas, Centris leprieuri e Centris sponsa, esta última com a menor frequência de visitas.
O mesmo autor relaciona o sucesso reprodutivo de $S$. paralias naquele local com a reprodução vegetativa e com o sistema autogâmico facultativo. Assim, mesmo recebendo poucas visitas das abelhas coletoras de óleo, o processo de polinização da espécie torna-se facilitado pela geitonogamia e/ou autopolinização.

Na espécie estudada, pode-se observar que ocorre grande variação no número de glândulas entre indivíduos na população, principalmente relacionada ao par 1 (sépala anterior), localizado sob o abdome do polinizador quando pousado na flor, tanto na espécie estudada quanto nas demais Malpighiaceae (Faegri \& van der Pijl 1979; Anderson 1979; Simpson \& Neff 1981; Sazima \& Sazima 1989; Vogel 1990; Gaglianone 2000; Teixeira \& Machado 2000; M.R. Sigrist, dados não publicados; C.B.N. Costa, dados não publicados). Vogel (1974 apud Anderson 1979) mencionou que muitos gêneros poderiam ter perdido as glândulas desta sépala, uma vez que a orientação da abelha e a restrição de coleta de óleo com as pernas dianteiras e medianas fazem as glândulas desta sépala inacessíveis. Isto ocorreria a menos que a abelha dê a volta na flor (Anderson 1979), comportamento que não tem sido observado nesta e em outras espécies de Malpighiaceae (Faegri \& van der Pijl 1979; Simpson \& Neff 1981; Sazima \& Sazima 1989; Vogel 1990; Gaglianone 2000; Teixeira \& Machado 2000; M.R. Sigrist, dados não publicados; C.B.N. Costa, dados não publicados). Desta forma, a não funcionalidade de algumas glândulas, acarretada pela incapacidade de utilização pelos polinizadores, pode ter possibilitado o aparecimento de morfos nas populações que não apresentam estas glândulas como um caráter adaptativo por gerar economia de recursos na produção de recompensa. Observações mais detalhadas sobre o comportamento dos polinizadores e dos outros visitantes florais são necessárias para confirmar esta hipótese, que infelizmente não puderam ser realizados devido à baixa freqüência das visitas na população escolhida.

Por outro lado, a ocorrência de variação dentro dos indivíduos e de indivíduos com nove glândulas, sem contudo haver constância na glândula presente no par 1 (esquerda ou direita), enfraquece a hipótese de simplesmente ocorrer morfos bem estabelecidos na população. A realização de cruzamentos experimentais entre os morfos e nas suas progênies pode ajudar a compreender a segregação e estabelecimento destes morfos na população.

Acredita-se que a hipótese de ocorrência de mimetismo entre flores com 10 glândulas e flores com oito glândulas pode ser desconsiderada para esta 
espécie, uma vez que os indivíduos que possuem esta pequena variação numérica continuam gastando energia para a produção da mesma quantidade de óleo, sendo então aparentemente um sistema pouco vantajoso do ponto de vista energético para a planta. Também não há engano do polinizador, pois ele aparentemente não busca utilizar as glândulas da sépala de número 1 nas flores que possuem 10 glândulas.

De acordo com os resultados, pode-se dizer que ocorre uma compensação na produção de óleo em flores com oito glândulas, ou seja, elas produzem praticamente a mesma quantidade de óleo que as flores com 10 glândulas. Esta hipótese é reforçada pelo fato de todos os pares das flores com 10 glândulas serem funcionais, conforme evidenciado pelo teste com Sudan IV. Dessa forma, os morfos com número inferior de glândulas fornecem mais recompensa aos polinizadores sem custo energético adicional, podendo ter seu sucesso reprodutivo incrementado, uma vez que provavelmente estes morfos seriam mais atrativos aos polinizadores. Este aumento do sucesso reprodutivo poderia ser confirmado por meio de estimativas deste, tais como pela taxa de frutificação.

\section{Agradecimentos}

Os autores agradecem à Dra. Ana Maria Giulietti e à Dra. Nádia Roque pelas sugestões nas versões iniciais do manuscrito; Eduardo L. Borba agradece ao CNPq pela concessão da bolsa de Produtividade em Pesquisa.

\section{Referências bibliográficas}

Anderson, W.R. 1979. Floral conservatism in neotropical Malpighiaceae. Biotropica 11: 219-223.

Barroso, G. M. 1991. Sistemática de Angiospermas do Brasil. v. 2. Viçosa, Universidade Federal de Viçosa.

Buchmann, S.L. 1987. The ecology of oil flowers and their bees. Annual Review of Ecology and Systematics 18: 343-369.

Faegri, K. \& van der Pijl, L. 1979. The principles of pollination ecology. Oxford, Pergamon Press.

Gaglianone, M.C. 2000. Interações de Epicharis (Apidae, Centridini) e flores de Malpighiaceae em um ecossistema de cerrado. Anais do IV Encontro de Abelhas (Ribeirão Preto): 246-252.

Gates, B. 1982. Banisteriopsis, Diplopterys (Malpighiaceae). Flora Neotropica monograph 30: 1-237.

Sazima, M. \& Sazima, I. 1989. Oil-gathering bees visit flowers of eglandular morphs of the oil-producing Malpighiaceae. Botanica Acta 102: 106-111.

Simpson, B.B. \& Neff, J.L. 1983. Evolution and diversity of floral rewards. Pp. 142-159. In: C.E. Jones \& R.J. Little (eds.). Handbook of experimental pollination biology. New York, Scientific and Academic Editions.

Teixeira, L.A.G. \& Machado, I.C. 2000. Sistema de polinização e reprodução de Byrsonima sericea DC. (Malpighiaceae). Acta Botanica Brasilica 14: 347-357.

Vinson, S.B.; Willians, H.J.; Frankie, G.W. \& Shrum, G. 1997. Floral lipid chemistry of Byrsonima crassifolia (Malpighiaceae) and the use of floral lipids by Centris bees (Hymenoptera-Apidae). Biotropica 29: 76-83.

Vogel, S. 1990. History of the Malpighiaceae in the light of pollination ecology. Memoirs of the New York Botanical Garden 55: 130-142. 